\title{
Diffused-Quantum-Well Vertical Cavity Fabry-Perot Reflection Modulator
}

\author{
Wallace C.H. Choy, S.F. Ip and E. Herbert Li \\ Department of Electrical and Electronic Engineering, \\ The University of Hong Kong, Pokfulam Road, Hong Kong
}

\begin{abstract}
This is a first report to use diffused quantum well (DFQW) as the active cavity of the Fabry-Perot reflection modulator. Apart from the simple fabrication process of the DFQW, this material system provides a wavelength tuning range and improves the modulation properties of the device which thus is competitive to the same kind of modulator.
\end{abstract}

\section{INTRODUCTION}

Currently, surface-normal Fabry-Perot (FP) etalon modulators based on III-V multiple quantum well (MQW) semiconductors for potential applications such as twodimensional arrays for interconnection, and optical processing have gained considerable interest. This type of modulator has the advantage of providing a large area in the coupling of light in and out of the device and is compatible with vertical opto-electronic integrating technology [1]. Moreover, this structure of modulator provides long effective optical interaction path and thus reduces the applied voltage swing [2]. In this work, annealing induced non-square diffused-QW (DFQW) structure[3] is first introduced as the intrinsic material in the asymmetric-FP vertical cavity so that the operation wavelength $\lambda_{\text {op }}$ of the modulator can be fine tuned by controlling the disordering of regular as-growth $\mathrm{QW}$. This structure can serve as a high performance FP modulator by improving the modulation properties and providing an operation wavelength tuning range. It is expected that this DFQWs based FP modulator will become an important candidate for the vertical integrating technology. The electro-optical effects of DFQW structure on the total reflectance $R_{\text {tot }}$ of the modulator are analyzed together with the figures of merit, change of $R_{\text {tot }}\left(\Delta R_{100}\right)$, and the ratio $\Delta R_{\text {tot }} / R_{\text {tot }}$.

\section{MODELING}

We consider the DFQW resonant-etalon reflection modulator structure in a simple way, as shown in Fig. 1(a), where the inner intrinsic DFQW consists of 61 periods of $101 \AA / 100 \AA \mathrm{Al}_{0.3} \mathrm{Ga}_{0.7} \mathrm{As} / \mathrm{GaAs}$. The extent of diffusion is characterized by a diffusion length $L_{d}\left(L_{d}=0\right.$ represents the as-growth $\mathrm{QW}$ ) [3]. The front and back quarter wavelength Bragg reflectors are alternative AlAs and $\mathrm{AlGaAs}$ layers with reflective indices taken from [4] which are $\mathrm{p}$ - and $\mathrm{n}$ - doped as in a p-i-n structure. The bottom reflectance $R_{B}$ is set at about 0.97 , the top one $R_{T}$ is calculated from the relation $R_{T}=R_{b} \exp \left(-2 \alpha_{c} l_{c}\right)$ where $l_{c}$ and $\alpha_{c}$ are the cavity length and absorption coefficient respectively, $\alpha_{c}$ is calculated from a model similar to [5] without the polarization sensitivity; the relation of $R_{T}$ is the impedance-matching condition for zero total reffectivity at resonance[2] so that the contract ratio $\mathrm{CR}$ of the modulation can become infinity. The reflectance of the FP modulator can be calculated by using the scattering matrices method as shown in Fig. 1b. The arrow indicates the direction of propagation of the field, where $F$ (forward) and B (backward) are the electric field of the light. The total reflectance is defined as $R_{t o t}$ $=\left(B_{1} / F_{1}\right)^{2}=\left|\left(s_{21}+R_{B 5}{ }^{1 / 2} s_{22}\right) /\left(s_{11}-R_{1 T}{ }^{1 / 3} S_{12}\right)\right|^{2}$, where $R_{B 5}$ and $R_{1 T}$ are the reflectance of the interface between $(B, 5)$ and between $(1, T)$ respectively, the negative sign of $R_{1 T}$ indicates that the refractive index of medium $T$ is higher than that of medium 1 since medium 1 is considered to be air $\left(n_{\mathrm{air}}=1\right)$, and $s_{\mathrm{ij}}$ are the matrix elements of a $2 \times 2$ scattering matrix $S$. $S$ is defined as $M_{T} \times M_{Q w} \times M_{B}$, where $M_{j}$ is the scattering matrix of region $j$ for $F_{j}=m_{j 11} F_{j+1}+$ $m_{j 12} B_{j+1}$ and $B_{j}=m_{j 21} F_{j+1}+m_{j 22} B_{j+1}, m_{j a b}$ are elements of $M_{j}$, and the subscripts $\mathrm{a}$ and $\mathrm{b}$ are integer 1 or 2 . It is assumed that the field in top and bottom reflectors (medium $\mathrm{T}$ and B) will only undergo phase change without any residence loss while the field in the intrinsic DFQW layer will involve both phase changes $\exp \left(i \delta_{c} l_{c}\right)$ and loss $\exp \left(-\alpha_{c} l_{c}\right)$. The phase change per unit length is defined as $\delta_{i}=2 \pi n_{i} / \lambda_{\text {op }}$ where $n_{i}$ and $\lambda_{\text {op }}$, are the refractive index of medium (i) and the operational wavelength, respectively.

\section{RESULTS}

It is interesting to note that the FP modulator can operate at FP mode for cases from $L_{d} 0$ to $40 \AA$ stepped by $10 \AA$ without any re-design of the active region of the modulator. However, since the operation wavelength will shift while the QW structure undergoes an interdiffusion, 
the top and bottom Bragg reflectors, the number of the alternative layers, and the thickness of each quarter wavelength layer have to be rearranged. The number of period of top and bottom reflectors are 8.5 and 16.5 of alternative $734 \AA$ (AlAs) and $639 \AA \quad\left(\mathrm{Al}_{0.3} \mathrm{Ga}_{0.7} \mathrm{As}\right.$ ) respectively when the cavity structure is as-growth square wells $\left(L_{d}=0\right)$. While the $Q W$ structure undergoes an interdiffusion to $L_{d}=40 \AA$, the top and bottom reflectors will be amended to 7.5 and 16.5 periods of alternative $633 \AA$ (AlAs) and $548 \AA\left(\mathrm{Al}_{0.3} \mathrm{Ga}_{0.7} \mathrm{As}\right)$ respectively. As a whole, the total thickness of the modulator will be reduced for different DFQW's cavities. Table 1 shows the modulation properties of the DFQWs modulators with an identical thickness of cavity. For the cases from $L_{d}=0$ to $20 \AA$, the change of reflectance $\left(\Delta R_{t a}\right)$ have roughly same value. Moreover, there are two possible $\lambda_{\text {op }}$ that the first $\lambda_{\text {op }}$ of $L_{d}=10$ will have relative low residence loss but high minimum value of $R_{w 0}, R_{w t}(\min )$, at FP mode while the second $\lambda_{o p}$ is just vice versa. The $R_{\text {tot }}(\mathrm{min})$ for the case $L_{d}=30 \AA$ is the lowest $\left(1.0418 \times 10^{-5}\right)$ with low residence loss. Although the $\Delta \mathrm{R}_{\text {tot }}$ is also reduced to minimum (0.100) when comparing with other $L_{d} s$ ' cases, the figure of the merit $\Delta R_{\text {tot }} / R_{\text {tot }}$ is extremely high about 9600 . If the interdiffusion is further increased to $L_{d}=40 \AA$, the $\lambda_{\text {op }}$ will further blue-shift [6] to $0.7645 \mu \mathrm{m}$. Also the applied electric field $F(\mathrm{kV} / \mathrm{cm})$ will reduce to $50-80 \mathrm{kV} / \mathrm{cm}$ though the $\mathrm{R}_{\mathrm{tot}}(\mathrm{min})$ become high. All in all, the same asgrowth QW cavity structure can operate between 0.8747 and $0.794 \mu \mathrm{m}$ under different interdiffusion from $L_{d}=0$ to $30 \AA$. If high $R_{\text {wo }}(\mathrm{min})$ is not an obstacle in an operation, the wavelength tuning range will increase to $(0.8747$ to $0.7645 \mu \mathrm{m}$ ) about $110 \mathrm{~nm}$.

In fact, the whole modulation will further improve by adjusting the cavity thickness $l_{c}$ for $n \pi$ phase variation of the field in different $\mathrm{L}_{d} s$ ' cases. In order to compare with the result of as-growth square $\mathrm{QW}$ modulator, the adjusted $l_{c}$ of different $L_{d} s$ ' cases is set nearest to the $l_{c}$ of the square QW modulator. Fig.2a and Fig.2b show the reflectance spectra of different applied field of the cases $\mathrm{L}_{\mathrm{d}}=0$ (square $\mathrm{QW}$ ) and $\mathrm{L}_{\mathrm{d}}=40 \AA$ respectively. For the case $L_{d}=0$, the OFF-state operated at $\lambda_{o p}=0.875 \mu \mathrm{m}$ with field $F=130 \mathrm{kV} / \mathrm{cm}$ while the On-state is with field $\mathrm{F}=0$. It is found that the $\lambda_{\text {op }}$ of FP mode of higher field operation will shift to shorter wavelength because the change of reflective index $\Delta \mathrm{n}$ of the DFQW cavity under reverse bias. The $\Delta \mathrm{n}$ is due to the Quantum Confined Stark Effect and can be calculated from the spectrum of the change of absorption coefficient by Kramer-Krönig relation. Moreover, for the spectra of the case $\mathrm{F}=0,50$ and 90 $\mathrm{kV} / \mathrm{cm}$, the reflectance are close together at the $\lambda_{\text {op. }}$. This can be explained by the packing of absorption spectra at the $\lambda_{o p}$ as shown in [7]. The $R_{T}$ is designed for zero $R_{t o t}$ at $F=130 \mathrm{kV} / \mathrm{cm}$ (OFF-state), so from figure 2 , the modulation is operated at the ON-OFF state with $\Delta R_{\text {tol }} / R_{\text {tot }}$ $=0.6427 / 3.313 \times 10^{-3}=194$. In Fig. $2 b$, the case of $L_{d}=$ $40 \AA$, the $\lambda_{\text {op }}$ is blue shift to $0.7727 \mu \mathrm{m}$. The FP resonance with zero reflectance is operated at $\mathrm{F}=80 \mathrm{kV} / \mathrm{cm}$, the $\Delta \mathrm{R}_{\text {to }}$ of $\mathrm{ON}(\mathrm{F}=0)$ and $\mathrm{OFF}(\mathrm{F}=80 \mathrm{kV} / \mathrm{cm})$ states is 0.6872 with extremely low $R_{\text {tox }}(\min )$ value $4.7633 \times 10^{-5}$. Thus the $\Delta R_{\text {wo }} / R_{\text {bot }}$ is as high as 15000 . This value is the highest from what we recognize in the published. Moreover, the applied field required for operation also reduces from $130 \mathrm{kV} / \mathrm{cm}$ at $\mathrm{L}_{\mathrm{d}}=0$ to $80 \mathrm{kV} / \mathrm{cm}$ in this case. This can therefore minimize the voltage swing. The results of other applied field are shown in Table 2. The $\Delta \mathrm{R}_{\mathrm{wt}}$ increases from $L_{d}=0$ to $L_{d}=20 \AA$ and then reduce to 0.4873 at $L_{d}=$ $40 \AA$. While the $R_{\text {to }}(\min )$ keeps very low and only within an acceptable fluctuation, the residence loss of different $\mathrm{L}_{d} \mathrm{~s}^{\prime}$ cases is just between 550 and $860 \mathrm{~cm}^{-1}$ except the second possible $\lambda_{\mathrm{op}}$ which can operate at low $\mathrm{R}_{\mathrm{tot}}(\mathrm{min})$, of $\mathrm{L}_{\mathrm{d}}=10 \AA$ with residence loss $1200 \mathrm{~cm}^{-1}$. Two outstanding cases $L_{d}=30$ and $40 \AA$ are mentioned that for the case $L_{d}$ $=30 \AA$, the residence loss reduces to lowest as well as low $\mathrm{R}_{\mathrm{tot}}(\mathrm{min}), 350 \mathrm{~cm}^{-1}$ and $2.327 \times 10^{-4}$ respectively. While for the case $L_{d}=40 \AA$, the applied field can be reduced to $80 \mathrm{kV} / \mathrm{cm}$ (lower power consumption) with extremely high $\Delta R_{t o} / R_{t+1} 15000$ as mentioned above. Together with the distinct feature of blue shift of $\lambda_{\mathrm{cp}}$ and simple thermal annealing processes for producing DFQW, this FP reflection modulator will become competitive in same kind of modulator.

\section{CONCLUSION}

In conclusion, we have analyzed the reflectivity property of a vertical cavity FP reflection modulator with different $L_{d}$ 's and its electro-optical effect. The blue shift of $\lambda_{\text {op }}$ is due to the effect of DFQW which suggests a wide band-width FP reflection modulator with $\Delta \lambda_{\text {op }} \cong$ $110 \mathrm{~nm}$. Moreover, the $\mathrm{L}_{\mathrm{d}}=30 \AA \mathrm{DFQW}$ modulator with adjusted $1_{c}$ can operate at lowest residence loss and low $R_{\omega t}(\mathrm{~min})$ implies highest figure of merit $\Delta \mathrm{R}_{\mathrm{t}} / \mathrm{R}_{\mathrm{wt}}$ while the case $L_{d}=40 \AA$, only $80 / 130 \times 100 \%=61 \%$ applied field is required and achieved highest $\Delta R_{\text {to }} / R_{\text {to }}=15000$. All these properties make the DFQWs vertical cavity modulator become competitive device.

\section{ACKNOWLEDGMENT}

This work is financially supported by the HKUCRCG grant.

\section{REFERENCE}

[1] G.A. Evan, et al., " Characteristics of Coherent TwoDimensional Grating Surface Emitting Diode Laser Arrays During CW Operation", IEEE J. of Quantum Electron. vol. 27, pp.1594-1605, 1991.

[2] K.K. Law, J.L. Merz, and L.A. Golden, "Superlattice Surface-Normal Asymmetric Fabry-Perot Reflection Modulators: Optical Modulation and Switching", J. of Quantum Electron. vol. 29, pp.727-739, 1993. 
[3] E.H. Li, B.L. Weiss and K.S. Chan, "Effect of interdiffusion in the subbands in an $\mathrm{Al}_{\mathrm{x}} \mathrm{Ga}_{1 \cdot \mathrm{x}} \mathrm{As} / \mathrm{GaAs}$ single-quantum-well structure", Phys. Rev. B, vol. 46, no.23, pp.15181-1.5192, 1993.

[4] B. Jensen and W. D. Jensen, "The Reflective Index Near the Fundamental Absorption Edge in $\mathrm{Al}_{\mathrm{x}} \mathrm{Ga}_{1-\mathrm{x}} \mathrm{As}$ Ternary Compound Semiconductors", IEEE J. of Quantum Electron. vol. 27, pp.40-44, 1991.

[5] E.H. Li, K.S. Chan, B.L. Weiss, and J. Micallef, "Quantum-confined Stark effect in interdiffused
AlGaAs/GaAs quantum well", Appl. Phys. Lett. vol. 63, pp.533-535, 1993.

[6] E.H. Li and W.C.H. Choy, " Electro-optical and Electroabsorptive Modulation Properties in Interdiffusion Modified AlGaAs/GaAs Quantum Wells", IEEE Photon. Technol. Lett. to be published.

[7] W.C.H. Choy and E.H. Li, IEEE, "High performance Diffused Quantum. Wells Fabry Perot reflection modulator", J. of Quantum Electron., submitted for published.

\begin{tabular}{ccccccc}
\hline $\mathrm{L}_{\mathrm{d}}(\AA)$ & $\lambda_{\mathrm{op}}(\mu \mathrm{m})$ & $\Delta \mathrm{R}_{\text {tot }}$ & $\mathrm{R}_{\text {tot }}(\mathrm{min})$ & $\begin{array}{c}\mathrm{F}(\mathrm{kV} / \mathrm{cm}) \\
{[\mathrm{ON}-\mathrm{STATE}]}\end{array}$ & $\begin{array}{c}\mathrm{F}(\mathrm{kV} / \mathrm{cm}) \\
\text { [OFF-STATE] }\end{array}$ & $\alpha_{\text {loss }}\left(\mathrm{cm}^{-1}\right)$ \\
\hline 0 & 0.8747 & 0.6427 & $3.3125 \times 10^{-3}$ & 0 & 130 & 550 \\
10 & 0.8564 & 0.6615 & 0.1628 & 0 & 130 & 860 \\
10 & 0.8522 & 0.6482 & 0.0892 & 0 & 130 & 1200 \\
20 & 0.8202 & 0.6899 & 0.00346 & 130 & 90 & 1090 \\
30 & 0.7940 & 0.1000 & $1.0418 \times 10^{-5}$ & 110 & 90 & 640 \\
40 & 0.7645 & 0.3150 & 0.585 & 80 & 50 & 1050 \\
\hline
\end{tabular}

Table 1. Features of the DFQW reflection modulators with an identical thickness of cavity.

\begin{tabular}{cccccccc}
\hline $\mathrm{L}_{\mathrm{d}}(\AA)$ & $\lambda_{\mathrm{op}}(\mu \mathrm{m})$ & $\Delta \mathrm{R}_{\text {tot }}$ & $\mathrm{R}_{\text {tot }}(\mathrm{min})$ & $\begin{array}{c}\mathrm{F}(\mathrm{kV} / \mathrm{cm}) \\
\text { [ON-STATE] }\end{array}$ & $\begin{array}{c}\mathrm{F}(\mathrm{kV} / \mathrm{cm}) \\
{[\text { OFF-STATE] }}\end{array}$ & $1_{\mathrm{c}}(\mu \mathrm{m})$ & $\begin{array}{c}\alpha_{\text {Ioss }} \\
\left(\mathrm{cm}^{-1}\right)\end{array}$ \\
\hline 0 & 0.8747 & 0.6427 & $3.313 \times 10^{-3}$ & 0 & 130 & 1.2273 & 550 \\
10 & 0.8564 & 0.7059 & $3.789 \times 10^{-2}$ & 0 & 130 & 1.2063 & 860 \\
10 & 0.8523 & 0.6300 & $6.694 \times 10^{-3}$ & 0 & 110 & 1.2063 & 1200 \\
20 & 0.8270 & 0.6612 & $2.050 \times 10^{-3}$ & 0 & 130 & 1.2817 & 738 \\
30 & 0.7991 & 0.5766 & $2.327 \times 10^{-4}$ & 0 & 110 & 1.2399 & 350 \\
40 & 0.7713 & 0.6872 & $4.763 \times 10^{-5}$ & 0 & 80 & 1.2844 & 691 \\
\hline
\end{tabular}

Table 2. Features of the DFQW reflection modulators with $1_{c}$ nearest to that of the square $Q W$ modulator.

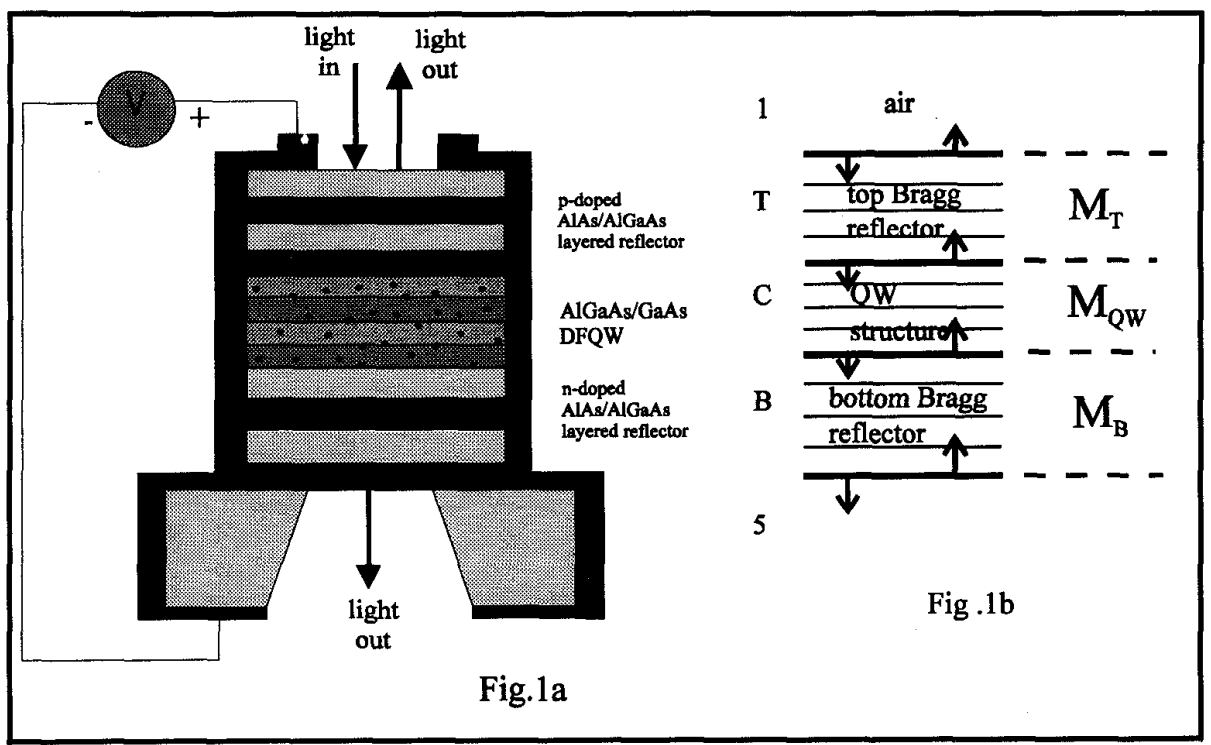

Fig. 1a Schematic crosssection of the threesections exptiaxial FebryPerot reflection modulator Fig. 1b Illustration of the scattering matrices in deriving design equations. 


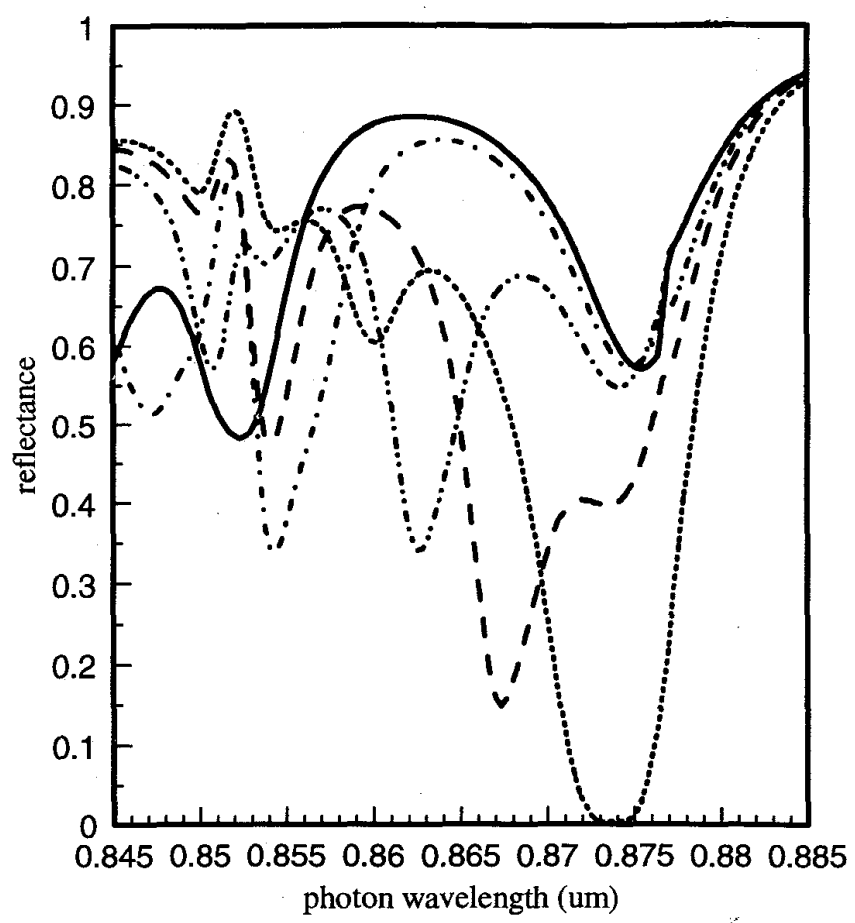

Fig.2a The reflectance spectra of $L d=0$ with field $F=0$ (soild), 50 (dash-dot), 90 (dash-double dot,), 110 (long-dash) and $130 \mathrm{kV} / \mathrm{cm}$ (dot).

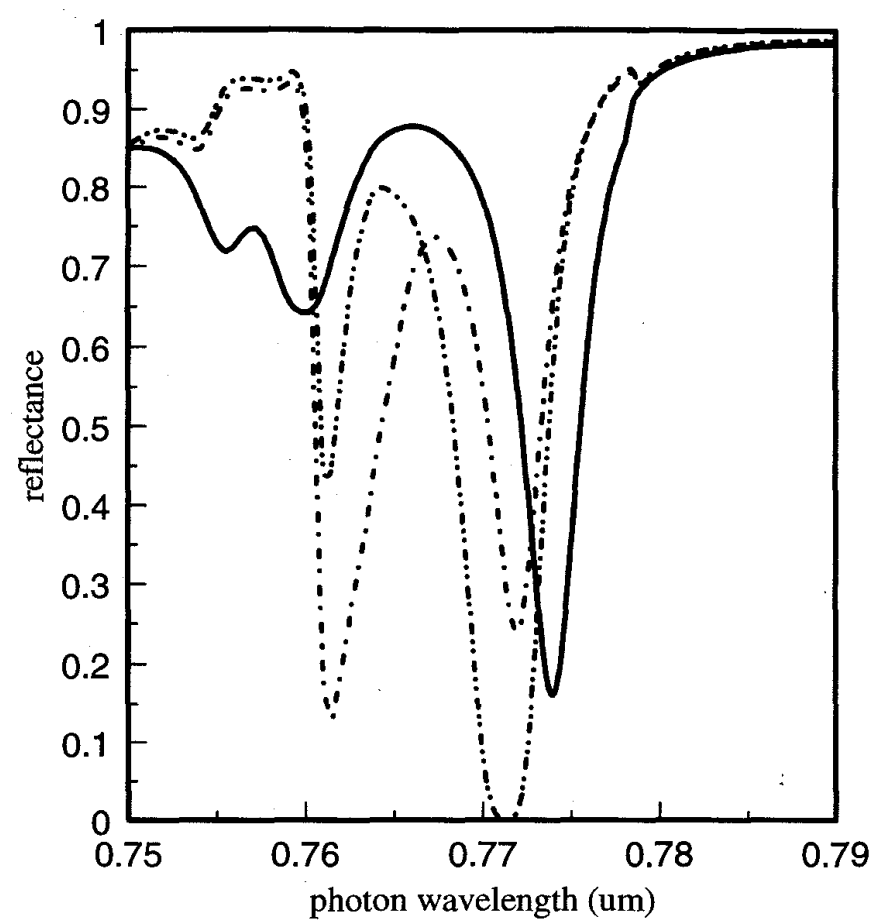

Fig. $2 b$ The reflectance spectra of $L d=40 \mathrm{~A}$ with field $\mathrm{F}=0$ (soild), 50 (dash-dot), 80 (dash-double dot). 